\title{
EL VERMICOMPOST: SU EFECTO EN ALGUNAS PROPIEDADES DEL SUELO Y LA RESPUESTA EN PLANTA ${ }^{1}$
}

\author{
Lolita Durán-Umaña ${ }^{2}$, Carlos Henríquez-Henríquez ${ }^{3}$
}

\begin{abstract}
RESUMEN
El vermicompost: su efecto en algunas propiedades del suelo y la respuesta en planta. Se estudió el efecto del vermicompost sobre algunas propiedades del suelo y la biomasa vegetal. En invernadero se mezcló vermicompost en proporciones de $0,25,50,75$ y $100 \%$ con un Andisol y un Ultisol en macetas de un litro y se sembró sorgo cuatro veces consecutivamente y por períodos de 45 días. Del 2003 al 2006, en un potrero sembrado con pasto estrella (Cynodon nlemfuensis) en un Inceptisol, se ubicaron parcelas de $25 \mathrm{~m}^{2}$ donde se aplicaron 0,10 y 20 t/ha/año de vermicompost. Se realizaron análisis de suelo y mediciones de la biomasa vegetal. En las macetas, la aplicación de vermicompost incrementó el P, Ca, Mg, K y la CICE. Aunque el pH aumentó también se observó un incremento en la acidez intercambiable lo cual pudo deberse a los iones hidronio de la materia orgánica aplicada. La mayor acumulación de biomasa se alcanzó en la proporción de $50 \%$ de vermicompost mientras que proporciones mayores provocaron una disminución. Se encontró un efecto diferencial por el tipo de suelo. A nivel de campo, el vermicompost no afectó la biomasa del C. nlemfuensis. No hubo efecto del vermicompost sobre las propiedades del suelo, lo cual pudo deberse a las propiedades químicas originales del mismo.
\end{abstract}

Palabras clave: Fertilidad de suelos, abono orgánico.

\begin{abstract}
Effect of vermicompost in some soil properties and plant response. The effect of vermicompost on some soil properties and on plant biomass was studied. In a greenhouse, different proportions of vermicompost $(0,25,50,75$ and $100 \%$ ) were mixed with an Andisol and Ultisol soils In 11 pots, and sorghum seeds were sown four consecutive times during 45 days. From 2003 to 2006 in a field sown with Cynodon nlenfluensis, $25 \mathrm{~m}^{2}$ plots were established, in which 0,10 and $20 \mathrm{t} / \mathrm{ha} /$ year of vermicompost were applied. Biomass accumulation and soil properties were evaluated. Vermicompost applied to pots elevated $\mathrm{P}, \mathrm{Ca}, \mathrm{Mg}, \mathrm{K}$ and CICE. Although soil $\mathrm{pH}$ also increased, exchangeable acidity increased probably as a consequence of hidronium ions released by the organic matter applied. The highest biomass accumulation was reached with a $50 \%$ proportion of vermicompost, while higher proportions decreased biomass accumulation. A differential effect due to soil type was observed. In the field, biomass of $C$. nlenfluensis was not affected by the application of the vermicompost. There was no effect of vermicompost on soil properties, which was probably due to the original properties of the soils tested.
\end{abstract}

Key words: Soil fertility, compost.

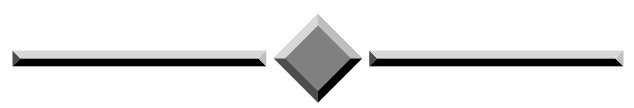

\section{INTRODUCCIÓN}

En el mercado existe una gran variedad de tipos de abonos orgánicos debido tanto a la cantidad de materias primas disponibles como a los diferentes procesos de elaboración (Soto 2003, Soto y Meléndez 2003). Esto ha motivado en cierta forma, que en los últimos años se incremente su utilización para la producción de muchos

\footnotetext{
1 Recibido: 18 de mayo, 2009. Aceptado: 17 de mayo, 2010. Datos parciales del Proyecto VI-510-A4-109 y VI-510-A8-320, financiados por la Vicerrectoría de Investigación de la Universidad de Costa Rica.

2 Sede del Atlántico de la Universidad de Costa Rica. Turrialba, Costa Rica. lolita.duran@ucr.ac.cr

3 Sede del Atlántico y Centro de Investigaciones Agronómicas de la Universidad de Costa Rica. San José, Costa Rica. carlos.henriquez@ucr. ac.cr
} 
cultivos (Meléndez 2003). Su demanda también se relaciona con el efecto positivo que estos materiales tienen sobre el mejoramiento de diversas propiedades del suelo así como por su posible uso como sustitutivos o complementos de los fertilizantes sintéticos (Bertsch 1998, Castro et al. 2009).

Debido a que los abonos orgánicos se catalogan como enmiendas o mejoradores del suelo, las dosis que usualmente se aplican son comparativamente más altas y variables que las utilizadas con los fertilizantes minerales (Bertsch 1998). Además las concentraciones de nutrimentos, usualmente son bajas cuando se toma en cuenta los kilogramos de nutrientes aplicados por cada tonelada de material (Castro et al. 2009). Otro estudio demostró como la materia prima utilizada tiene un impacto directo sobre la concentración y el comportamiento final de los nutrientes en vermicompostes (Durán y Henríquez 2007). Con base en lo anterior, es de esperar que la calidad final de estos productos sea muy diversa así como su efecto en el suelo y los cultivos luego de su aplicación (Meléndez 2003).

Castellanos y Pratt (1981) y Hartz et al. (2000), mencionan que el aporte de $\mathrm{N}$ y $\mathrm{C}$ al suelo puede ser muy significativo; y se favorece la humificación y la formación de estructuras que generen capacidad de carga a nivel coloidal. Además de $\mathrm{N}$ y $\mathrm{C}$, el aporte de otros elementos como el $\mathrm{Ca}, \mathrm{Mg}, \mathrm{K}$ y P puede ser importante, en suelos con una baja fertilidad (Evanylo et al. 2008). Algunos estudios han corroborado el efecto positivo de estos materiales en el mejoramiento de la capacidad buffer del suelo y su efecto en la disminución de la acidez y correspondiente aumento en el pH del mismo (Babou et al. 2007, Narambuye y Haynes 2007).

Es necesario generar más información que reúna en forma sistemática, los efectos de estos materiales sobre las propiedades del suelo así como su respuesta en planta. Este estudio tuvo como objetivo determinar el efecto de vermicompost sobre algunas propiedades químicas del suelo y en Cynodon nlemfuensis.

\section{MATERIALES Y MÉTODOS}

\section{Material}

Para la realización de este estudio se utilizó un vermicompost producido a partir de estiércol vacuno y elaborado en las instalaciones del Módulo Lechero ubicado en el Recinto de Turrialba, de la Universidad de Costa Rica. El proceso de fabricación de este vermicompost tuvo cinco fases que incluyeron el precompostaje inicial del estiércol, el proceso de conversión con la lombriz Eisenia fetida (conocida como "lombriz roja californiana"), la separación de la lombriz del material, un período de estabilización y finalmente el secado y cribado final.

\section{Análisis de laboratorio}

El vermicompost fue analizado con base en el análisis de contenido total de nutrimentos en base seca. El $\mathrm{N}$ y el $\mathrm{P}$ fueron determinados por colorimetría en tanto que $\mathrm{Ca}, \mathrm{Mg}$, $\mathrm{K}$ y elementos menores por absorción atómica. Adicionalmente se determinó el contenido de humedad y el $\mathrm{pH}$ en agua con pasta saturada (Sweizer et al. 2003).

Las muestras de suelo por otro lado, fueron analizadas de acuerdo a la metodología presentada por Henríquez y Cabalceta (1999) mediante la utilización de las soluciones extractoras Olsen Modificado y $\mathrm{KCl}$ $1 \mathrm{M}$ en una proporción 1:10. Los elementos $\mathrm{Ca}, \mathrm{Mg}, \mathrm{K}$, $\mathrm{Fe}, \mathrm{Mn}, \mathrm{Cu}$ y $\mathrm{Zn}$ fueron determinados por absorción atómica y $\mathrm{P}$ colorimétricamente. El $\mathrm{pH}$ fue determinado en agua a una relación 1:2,5 suelo solución y la acidez intercambiable extaída con $\mathrm{KCl} 1 \mathrm{M}$ en forma titrimétrica.

Los suelos utilizados en la fase de invernadero, fueron analizados al inicio del experimento y luego al finalizar las cuatro cosechas realizadas. En el caso del suelo del experimento de campo, éste fue muestreado y analizado al inicio (2003) y en dos ocasiones más (2004 y 2006).

\section{Área de experimentación}

El experimento constó de dos ensayos independientes, uno a nivel de invernadero y otro a nivel de campo, esto con el fin de evaluar el efecto del vermicompost bajo dos ambientes diferentes: una con condiciones controladas en un ensayo de corta duración (180 días) y otro a nivel de campo con un suelo de mayor fertilidad y a más largo plazo

\section{Ensayo de invernadero}

Para la fase de invernadero se utilizaron dos suelos de la provincia de Cartago: Andisol (de la localidad de Juan Viñas) y Ultisol (de la localidad de Paraíso). 
Los tratamientos consistieron en proporciones de 0 , $25,50,75$ y $100 \%$ vermicompost y suelo. Las mezclas fueron colocadas en potes de un litro de capacidad y dispuestas en invernadero en un diseño irrestricto al azar con cuatro repeticiones.

En los potes se sembraron ocho plantas de sorgo (Sorghum bicolor), por 45 días, luego de los cuales se extrajeron de las macetas con el fin de evaluar su biomasa total, separadas en su parte aérea y radical, secada a $60{ }^{\circ} \mathrm{C}$ y finalmente pesadas. Se procedió a sembrar nuevamente en el mismo sustrato, procedimiento que se realizó por cuatro ocasiones y en forma sucesiva sobre los mismos potes. Al final del experimento se tomaron muestras compuestas de suelo con el fin de analizar las características químicas finales. Este experimento fue realizado durante el año 2006.

Al final de la primera cosecha, las plantas de sorgo se extrajeron en forma completa de la maceta, separadas en su parte aérea y radical. La biomasa fue secada a $60^{\circ} \mathrm{C}$ y finalmente pesadas. El sustrato de los tratamientos fue luego homogenizado y vuelto a poner nuevamente en la maceta para volverlo a sembrar. Este procedimiento fue repetido hasta completar las cuatro cosechas.

\section{Ensayo de campo}

El ensayo de campo estuvo ubicado en el Módulo Lechero del recinto de Turrialba de la Sede del Atlántico, de la Universidad de Costa Rica en la zona de vida del bosque muy húmedo tropical premontano a 700 msnm (Holdridge 1978). La zona tiene una precipitación y temperatura promedio de $2649,9 \mathrm{~mm}$ y $21,8^{\circ} \mathrm{C}$ respectivamente y es dominada por Inceptisoles de origen aluvial de fertilidad alta. Para ello se eligió un potrero sembrado con pasto estrella (Cynodon nlemfuensis) por más de 25 años y en el cual se instalaron parcelas de $25 \mathrm{~m}^{2}$.
Los tratamientos consistieron en 0,10 y 20 t/ha/año de vermicompost el cual fue aplicado en cada uno de los cuatro años (del año 2003 al 2006) que duró el ensayo hasta alcanzar un acumulado de 0,40 y $80 \mathrm{t} / \mathrm{ha}$ al final del experimento. Los tratamientos tuvieron cuatro repeticiones y fueron dispuestos en un diseño de Bloques Completos al Azar (BCA). Para medir la respuesta en biomasa del pasto estrella en respuesta a la aplicación de vermicompost, se hicieron muestreos con cuadrículas de $0,5 \times 0,5 \mathrm{~m}$ y se procedió a cortar la biomasa aérea del pasto dentro del cuadro, secada en una estufa a $60{ }^{\circ} \mathrm{C}$ y finalmente pesada. Adicionalmente, en el cuarto muestreo se procedió también a evaluar el área completa de las parcelas $\left(25 \mathrm{~m}^{2}\right)$ con el fin de validar los datos obtenidos a partir de los muestreos, lo cual mostró estimados satisfactorios y congruentes entre las dos áreas de muestreo. También se procedió a hacer muestreos anuales de suelo (del 2003 al 2006), lo cual se realizó a través de muestras compuestas dentro de cada parcela.

El suelo fue analizado de acuerdo a la metodología explicada anteriormente en la parte de análisis de laboratorio (Henríquez y Cabalceta 1999).

\section{RESULTADOS Y DISCUSIÓN}

Los contenidos totales de elementos del vermicompost utilizado en este estudio (Cuadro 1), se consideran dentro del ámbito normal para este tipo de materiales según diversos autores (Schweizer et al. 2003, Soto y Meléndez 2003, Sullivan y Miller 2001). El pH fue de 7, lo cual está documentado como un valor esperable en abonos orgánicos estabilizados y maduros. En particular los contenidos de $\mathrm{N}$ y P fueron mayores que el promedio en comparación a otros tipos de abonos orgánicos (Wu y Martínez 2000).

El vermicompost utilizado mostró una consistencia suelta y friable a pesar de su contenido de humedad que

Cuadro 1. Caracterización química y humedad del vermicompost utilizado en el experimento. Turrialba, Costa Rica. 2006.

\begin{tabular}{|c|c|c|c|c|c|c|c|c|c|c|c|c|}
\hline \multirow{3}{*}{$\begin{array}{c}\text { Humedad } \\
\%\end{array}$} & \multirow[b]{3}{*}{ pH } & \multicolumn{11}{|c|}{ Contenido de minerales } \\
\hline & & \multicolumn{6}{|c|}{$\%$} & \multicolumn{5}{|c|}{$\mathrm{mg} / \mathrm{kg}$} \\
\hline & & $\mathbf{N}$ & $\mathbf{P}$ & $\mathbf{C a}$ & Mg & $\mathbf{K}$ & $\mathbf{S}$ & $\mathbf{F e}$ & $\mathrm{Cu}$ & $\mathbf{Z n}$ & Mn & B \\
\hline 68 & 7 & 2,35 & 1,88 & 3,34 & 0,78 & 0,30 & 0,46 & 5149 & 181 & 813 & 633 & 25 \\
\hline
\end{tabular}


fue del 68\% (P/P); se deduce que este comportamiento se debe a su alta capacidad de retención de humedad, lo cual está reportado para este tipo de materiales (Martínez 1996).

Con base en los contenidos presentados en el Cuadro 1 y el estimado de materia seca (32\%), se estimó que luego de cuatro años y tomando de referencia la mayor dosis acumulada de 80 t/ha, se aplicó el equivalente a $600 \mathrm{~kg} / \mathrm{ha}$ de $\mathrm{N}, 481 \mathrm{~kg} / \mathrm{ha}$ de P y $76 \mathrm{~kg} / \mathrm{ha}$ de K. Es importante mencionar que datos presentados por Castro et al. (2009) y Soto (2003), demuestran que no todo el $100 \%$ del total de elementos contenidos en los abonos orgánicos es disponible a corto plazo, para lo cual se estiman usualmente porcentajes menores al $25 \%$ de disponibilidad.

Fase de invernadero: Efecto en las propiedades químicas del suelo

Los resultados del análisis químico del Ultisol (suelo rojo) y Andisol (suelo volcánico) que fueron utilizados para la etapa de invernadero, se muestran en el Cuadro 2.
Con base en los niveles críticos para suelos (N.C.), a excepción del $\mathrm{K}$ en el Andisol (suelo volcánico en el Cuadro 2), los contenidos de $\mathrm{Ca}, \mathrm{Mg}$, $\mathrm{K}$ y P estuvieron por debajo de los niveles críticos. La Capacidad de Intercambio Catiónica Efectiva (CICE) de ambos suelos fue baja, aunque el pH fue levemente ácido. Todo lo anterior define ambos suelos con una fertilidad baja.

Aunque la evaluación de la biomasa se realizó en ambos suelos, por razones externas se pudo utilizar solo el Ultisol (suelo rojo en el Cuadro 2), para realizar el monitoreo final a través del análisis químico final, datos que son presentados en el Cuadro 3.

Elincremento en las proporciones de vermicompost produjo un aumento sustancial en los contenidos disponibles de $\mathrm{Ca}, \mathrm{Mg}, \mathrm{K}$ y $\mathrm{P}$ en forma lineal y sostenida (Cuadro 3). Este comportamiento está reportado por Castro et al. (2009) para diversos tipos de abonos orgánicos, aduciendo el aporte masivo de nutrientes en las altas cantidades de abonos orgánicos aplicados. Como puede observarse, aquí se excluye el tratamiento $100 \%$ de vermicompost, debido a que éste no se analizó por la metodología de extracción de

Cuadro 2. Caracterización química de los suelos utilizados en la fase de invernadero. Turrialba, Costa Rica. 2006.

\begin{tabular}{|c|c|c|c|c|c|c|c|c|c|c|c|c|}
\hline \multirow[b]{3}{*}{ Suelos } & \multirow[b]{3}{*}{ pH } & \multicolumn{11}{|c|}{ Contenido de minerales } \\
\hline & & \multicolumn{5}{|c|}{$\operatorname{cmol}(+) / l$} & \multicolumn{2}{|l|}{$\%$} & \multicolumn{3}{|c|}{$\mathrm{mg} / \mathrm{kg}$} & \multirow[b]{2}{*}{ Mn } \\
\hline & & Acidez & $\mathbf{C a}$ & Mg & $\mathbf{K}$ & CICE & SA & $\mathbf{P}$ & $\mathbf{Z n}$ & $\mathbf{C u}$ & $\mathbf{F e}$ & \\
\hline Ultisol & 5,3 & 0,10 & 2,92 & 0,85 & 0,08 & 3,95 & 3 & 5 & 3,6 & 16 & 60 & 25 \\
\hline Andisol & 5,0 & 0,75 & 2,42 & 0,60 & 0,29 & 4,06 & 18 & 4 & 2,5 & 10 & 108 & 13 \\
\hline Niveles críticos (Bertsch 1998) & 5,5 & 0,5 & 4 & 1 & 0,20 & 5 & 10 & 10 & 3 & 1 & 10 & 5 \\
\hline
\end{tabular}

Cuadro 3. Caracterización química de los sustratos provenientes de la mezcla de vermicompost y un Ultisol (suelo rojo) utilizado en la fase de invernadero luego de la cuarta siembra y extracción de sorgo a los 45 días después de la siembra. Turrialba, Costa Rica. 2006.

\begin{tabular}{|c|c|c|c|c|c|c|c|c|c|c|c|c|}
\hline \multirow[b]{3}{*}{ Tratamiento } & \multirow[b]{3}{*}{ pH } & \multicolumn{11}{|c|}{ Contenido de minerales } \\
\hline & & \multicolumn{5}{|c|}{$\operatorname{cmol}(+) / 1$} & \multicolumn{2}{|c|}{$\%$} & \multicolumn{3}{|c|}{$\mathrm{mg} / \mathrm{kg}$} & \multirow[b]{2}{*}{ Mn } \\
\hline & & Acidez & $\mathbf{C a}$ & Mg & $\mathbf{K}$ & CICE & $\mathbf{S A}$ & $\mathbf{P}$ & $\mathbf{Z n}$ & $\mathbf{C u}$ & $\mathbf{F e}$ & \\
\hline $0 \%$ Vermi & 5,0 & 0,11 & 3,32 & 1,26 & 0,12 & 4,81 & 2 & 3 & 3 & 18 & 30 & 19 \\
\hline $25 \%$ Vermi & 6,6 & 0,11 & 8,79 & 3,70 & 1,33 & 13,93 & 1 & 149 & 27 & 21 & 29 & 5 \\
\hline $50 \%$ Vermi & 6,9 & 0,36 & 11,10 & 6,97 & 2,32 & 20,64 & 1 & 142 & 55 & 17 & 32 & 9 \\
\hline $75 \%$ Vermi & 7,0 & 0,44 & 11,58 & 10,50 & 2,53 & 25,05 & 2 & 158 & 67 & 9 & 30 & 11 \\
\hline Niveles críticos (Bertsch 1998) & 5,5 & 0,5 & 4 & 1 & 0,20 & 5 & 10 & 10 & 3 & 1 & 10 & 5 \\
\hline
\end{tabular}


rutina la cual es exclusiva para suelos y por lo tanto los valores no son comparables.

Hubo efecto del vermicompost sobre el $\mathrm{pH}$ del suelo. Este valor se incrementó al aumentar las proporciones con vermicompost; en particular el tratamiento de $75 \%$ de vermicompost el cual mostró un valor de 7 . Pese a lo anterior, la acidez intercambiable también aumentó, lo cual puede deberse a una mayor cantidad de iones hidronios disponibles y provenientes de la ionización de los diferentes radicales presentes en la materia orgánica del vermicompost. A pesar de este incremento en la acidez intercambiable no afectó el porcentaje de saturación de acidez no se vio afectado, debido a que igualmente aumentaron las bases del suelo ( $\mathrm{Ca}, \mathrm{Mg}$ y K).

Con relación a lo anterior, Babou et al. (2007) y Naramabuye y Haynes (2007) encontraron que al adicionar abono orgánico en el suelo, se observó un aumento en la capacidad buffer del suelo. Datos de estos mismos autores refieren que las variables de acidez en el suelo tales como aluminio intercambiable y $\mathrm{pH}$ también fueron mejorados, pero difieren a los encontrados en este estudio, particularmente con relación a la acidez intercambiable.

Fase de invernadero: Efecto en la acumulación de biomasa de sorgo

Los datos de acumulación de biomasa de sorgo presentados en el Cuadro 4, muestran que tanto en el Ultisol como el Andisol, la aplicación de vermicompost tuvo un efecto positivo en el crecimiento de sorgo, la cual fue utilizada como planta indicadora. Como se comentó anteriormente, ambos suelos presentaron una fertilidad baja, por lo que era muy probable una respuesta vegetal positiva al mejoramiento de las propiedades del sustrato.

Al aplicar el vermicompost no solo se aportó nutrientes fundamentales para el crecimiento de la planta (N y $\mathrm{P}$ y otros elementos), sino también se mejoró la capacidad de carga de los suelos (Bertsch 1998, Naramabuye y Haynes 2007). Esto provoca un efecto positivo al estimular el crecimiento de las plantas (Chaimsohn et al. 2007).

La misma tendencia se observó al evaluar el porcentaje de materia seca que se presenta en el Cuadro 5. Las mayores respuestas en planta, se encontraron en las proporciones entre 25 y $50 \%$ de vermicompost (Cuadro 4 y 5). Proporciones mayores a $50 \%$, mostraron más bien una disminución en crecimiento (a excepción de la segunda cosecha en el suelo Ultisol, Cuadro 4). Esto confirma los resultados de Castro et al. (2009), en donde se encontró que la utilización del vermicompost puro o bien de proporciones muy altas, puede tener un efecto negativo en el crecimiento vegetal.

En términos de acumulación de biomasa, se observó una respuesta diferencial entre los dos tipos de suelos, aspecto que es observado también en la Figura 1 y el Cuadro 4. El Andisol tuvo una mayor respuesta en crecimiento. Es posible que el efecto de la materia orgánica aplicada por medio del vermicompost, provoque una dinámica diferencial de acuerdo al tipo de sistema de intercambio y a la capacidad buffer inicial del suelo. Es así como la materia orgánica aplicada en el Ultisol

Cuadro 4. Biomasa de sorgo expresada en peso seco promedio por planta ( $\mathrm{g}$ ) para cada una de las cosechas y de acuerdo a las proporciones de vermicompost utilizadas. Turrialba, Costa Rica. 2006.

\begin{tabular}{ccccc|cccc}
\hline Tratamiento & \multicolumn{4}{c}{ Ultisol } & \multicolumn{4}{c}{ Andisol } \\
\hline \multicolumn{1}{c}{ Cosechas* } & I & II & III & IV & I & II & III & IV \\
\hline $0 \%$ Vermi & $0,08 \mathrm{a}^{* *}$ & $0,06 \mathrm{a}$ & $0,07 \mathrm{a}$ & $0,16 \mathrm{a}$ & $0,15 \mathrm{a}$ & $0,10 \mathrm{a}$ & $0,15 \mathrm{a}$ & $0,30 \mathrm{a}$ \\
$25 \%$ Vermi & $0,89 \mathrm{~b}$ & $0,25 \mathrm{ab}$ & $0,47 \mathrm{~d}$ & $0,59 \mathrm{~b}$ & $1,25 \mathrm{~b}$ & $1,04 \mathrm{~b}$ & $0,49 \mathrm{~b}$ & $0,74 \mathrm{~b}$ \\
$50 \%$ Vermi & $0,64 \mathrm{~b}$ & $0,55 \mathrm{abc}$ & $0,44 \mathrm{~cd}$ & $0,67 \mathrm{~b}$ & $1,30 \mathrm{~b}$ & $1,48 \mathrm{~b}$ & $0,91 \mathrm{c}$ & $0,98 \mathrm{~b}$ \\
$75 \%$ Vermi & $0,59 \mathrm{~b}$ & $0,62 \mathrm{bc}$ & $0,21 \mathrm{ab}$ & $0,76 \mathrm{~b}$ & $1,14 \mathrm{~b}$ & $0,99 \mathrm{~b}$ & $0,51 \mathrm{~b}$ & $0,28 \mathrm{a}$ \\
$100 \%$ Vermi & $0,55 \mathrm{~b}$ & $0,86 \mathrm{c}$ & $0,30 \mathrm{bc}$ & $0,60 \mathrm{~b}$ & $0,55 \mathrm{a}$ & $0,86 \mathrm{ab}$ & $0,30 \mathrm{a}$ & $0,60 \mathrm{ab}$ \\
\hline
\end{tabular}

* Cosechas efectuadas en el mismo sustrato y en forma sucesiva en los mismos potes.

** Columnas con diferente letra son significativamente diferentes de acuerdo al análisis Tuckey-Kramer HSD al 0.05 . 
Cuadro 5. Porcentaje de materia seca promedio por planta de sorgo en cada una de las cosechas y de acuerdo a las proporciones de vermicompost utilizadas. Turrialba, Costa Rica. 2006.

\begin{tabular}{ccccc|cccc}
\hline Tratamiento & \multicolumn{4}{c|}{ Ultisol } & \multicolumn{4}{c}{ Andisol } \\
\hline \multicolumn{1}{c}{ Cosechas* } & I & II & III & IV & I & II & III & IV \\
\hline $0 \%$ Vermi & $9,09 \mathrm{a}^{* *}$ & $6,85 \mathrm{a}$ & $10,32 \mathrm{a}$ & $16,96 \mathrm{a}$ & $8,01 \mathrm{a}$ & 7,87 & $10,32 \mathrm{a}$ & $25,93 \mathrm{~b}$ \\
$25 \%$ Vermi & $12,75 \mathrm{c}$ & $11,98 \mathrm{~b}$ & $11,35 \mathrm{abc}$ & $22,15 \mathrm{~b}$ & $12,76 \mathrm{c}$ & 13,01 & $13,64 \mathrm{~b}$ & $25,20 \mathrm{~b}$ \\
$50 \%$ Vermi & $13,58 \mathrm{c}$ & $11,54 \mathrm{ab}$ & $12,87 \mathrm{bc}$ & $19,85 \mathrm{a}$ & $11,82 \mathrm{bc}$ & 12,61 & $15,34 \mathrm{~b}$ & $25,29 \mathrm{~b}$ \\
$75 \%$ Vermi & $12,57 \mathrm{bc}$ & $10,06 \mathrm{ab}$ & $13,43 \mathrm{c}$ & $19,86 \mathrm{a}$ & $11,47 \mathrm{bc}$ & 19,31 & $13,80 \mathrm{~b}$ & $19,29 \mathrm{a}$ \\
$100 \%$ Vermi & $9,73 \mathrm{ab}$ & $12,13 \mathrm{~b}$ & $10,91 \mathrm{ab}$ & $16,98 \mathrm{a}$ & $9,73 \mathrm{ab}$ & 12,13 & $10,91 \mathrm{a}$ & $16,94 \mathrm{a}$ \\
\hline
\end{tabular}

* Cosechas efectuadas en el mismo sustrato y en forma sucesiva en los mismos potes.

** Columnas con diferente letra son significativamente diferentes de acuerdo al análisis Tuckey-Kramer HSD al 0,05.

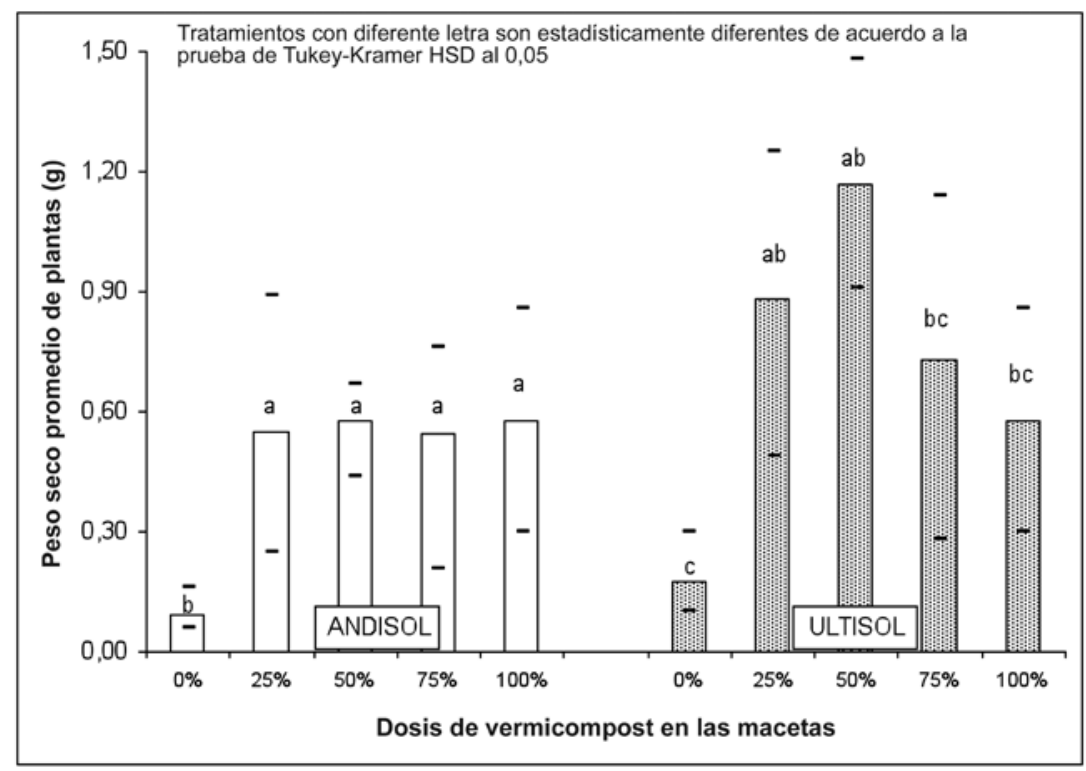

Figura 1. Peso seco promedio por planta en relación a diferentes proporciones de vermicompost en dos tipos de suelos utilizando sorgo como planta indicadora. Turrialba 2006.

(el cual se caracterizan por poseer sistemas de cambio caoliníticos y oxídicos con baja capacidad buffer), puede cumplir funciones tanto para suplir estabilidad química en sistema arcilloso al mismo tiempo que provee nutrientes al corto plazo (Babou et al. 2007).

De los datos anteriores se deduce que la respuesta a tasas similares de aplicación de vermicompost, puede variar sustancialmente de acuerdo al tipo de suelo, lo cual impide hacer estimaciones generalizadas al respecto.

Se eligieron los tratamientos 0 y $50 \%$ de vermicompost para poder representar mejor los comportamientos extremos con relación a esta variable (Figura 2). En esta figura se puede observar nuevamente el efecto diferenciado en la respuesta obtenida entre los dos tipos de suelo. 


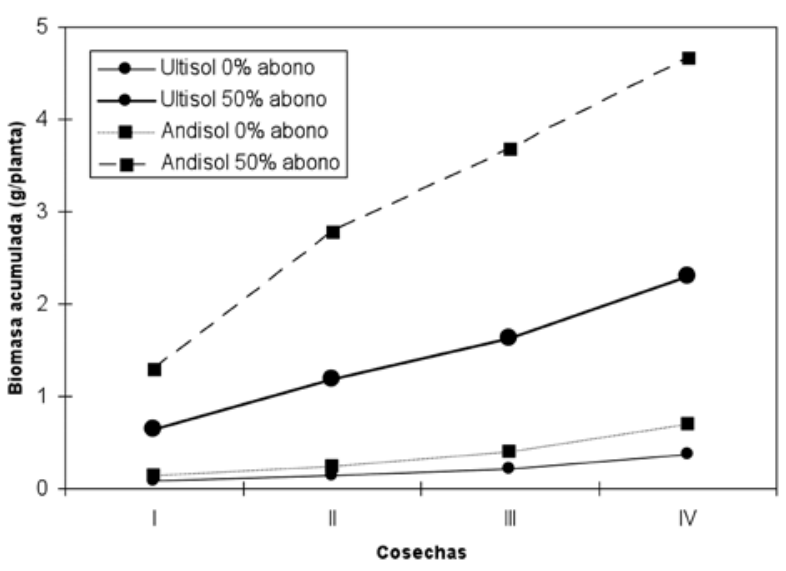

Figura 2. Representación de la acumulación de biomasa seca por planta de sorgo en cuatro cosechas en dos suelos con $50 \%$ de vermicompost y sin aplicación de una proporción de $50 \%$ de vermicompost. Turrialba, Costa Rica. 2006.

Lo que si es evidente en ambas figuras es el efecto positivo que tuvo el vermicompost en la acumulación de biomasa vegetal a través de todo el experimento.

\section{Fase de campo}

En el Cuadro 6 se presentan los resultados del análisis químico de suelo del lugar donde estuvo instalado el ensayo a través de los cuatro años de evaluación. En el mismo se presenta el resultado inicial así como los obtenidos en los muestreos a los dos y cuatro años. Es importante aclarar que en el cuarto año, el acumulado final en los tratamientos fue 0,40 y 80 t/ha.

El suelo no presentó limitantes de fertilidad (Cuadro 6). Esta es una posible explicación del porqué no se logró encontrar una respuesta significativa en los cambios en las propiedades de fertilidad ni tampoco una diferencia estadísticamente significativa (aunque si en tendencia) en la respuesta en biomasa del pasto estrella (Cynodon nlemfuensis), datos que son presentados en el Cuadro 7.

En el Cuadro 7 se presentan los estimados de biomasa por hectárea de pasto estrella por cada año de evaluación y en forma acumulada en cuatro años de estudio. Como se mencionó anteriormente, aunque las diferencias no fueron significativas, se encontró una tendencia de aumento entre el $0 \mathrm{y}$ el tratamiento de $40 \mathrm{t}$ acumuladas/año, con más de $500 \mathrm{~kg}$ de biomasa/ha sobre el tratamiento sin vermicompost. Estos datos podrían sugerir que el efecto de este tipo de abonos, en algunos casos se podrían estar manifestando en períodos largos de tiempo; ello implica que pueden no estarse observando respuestas en años individuales. Los autores sugieren que sería conveniente realizar en el futuro, experimentos con un mayor número de tratamientos y de repeticiones, por períodos de tiempo mayores.

Con relación al porcentaje de materia seca en pasto estrella, se encontró que el mejor tratamiento fue la

Cuadro 6. Caracterización química del suelo del experimento de campo ubicado en el Módulo Lechero de la Sede del Atlántico en Turrialba. Turrialba, Costa Rica. 2003-2006.

\begin{tabular}{|c|c|c|c|c|c|c|c|c|c|c|c|c|c|}
\hline \multirow{4}{*}{$\begin{array}{l}\text { Tratamientos } \\
\text { Año }\end{array}$} & \multirow{4}{*}{$\begin{array}{l}\text { Nivel } \\
\text { crítico }\end{array}$} & \multirow{3}{*}{$\frac{\mathrm{pH}}{\mathrm{H}_{2} \mathrm{O}}$} & \multicolumn{11}{|c|}{ Contenido de minerales } \\
\hline & & & \multicolumn{5}{|c|}{$\operatorname{cmol}(+) / 1$} & \multirow{2}{*}{$\%$} & \multicolumn{5}{|c|}{$\mathrm{mg} / \mathrm{l}$} \\
\hline & & & Acidez & $\mathbf{C a}$ & Mg & $\mathbf{K}$ & CICE & & $\mathbf{P}$ & $\mathbf{Z n}$ & $\mathbf{C u}$ & $\mathbf{F e}$ & Mn \\
\hline & & $<5,5$ & $>0,5$ & $<4$ & $<1$ & $<0,2$ & $<5$ & & $<10$ & $<3$ & $<1$ & $<10$ & $<5$ \\
\hline Inicial & & 5,7 & 0,29 & 11,13 & 4,11 & 1,48 & 17,0 & $2 \%$ & 59 & 9,0 & 32 & 339 & 9 \\
\hline \multirow[t]{3}{*}{ Año 2} & $0 \mathrm{t}$ & 5,7 & 0,2 & 12,6 & 4,0 & 1,1 & 17,9 & $1 \%$ & 62 & 10,1 & 33 & 510 & 15 \\
\hline & $10 \mathrm{t}$ & 5,9 & 0,2 & 13,3 & 4,8 & 1,4 & 19,7 & $1 \%$ & 73 & 9,3 & 26 & 411 & 14 \\
\hline & $20 \mathrm{t}$ & 5,6 & 0,2 & 11,9 & 4,1 & 1,3 & 17,5 & $1 \%$ & 67 & 9,9 & 30 & 456 & 15 \\
\hline \multirow[t]{3}{*}{ Año 4} & $0 \mathrm{t}$ & 5,6 & 0,1 & 11,1 & 4,1 & 1,5 & 16,8 & $1 \%$ & 53 & 7,8 & 26 & 230 & 11 \\
\hline & $10 \mathrm{t}$ & 5,5 & 0,1 & 10,8 & 3,9 & 1,4 & 16,3 & $1 \%$ & 52 & 7,4 & 22 & 203 & 9 \\
\hline & $20 \mathrm{t}$ & 5,6 & 0,1 & 10,8 & 4,0 & 1,8 & 16,8 & $1 \%$ & 52 & 7,8 & 26 & 188 & 11 \\
\hline
\end{tabular}


Cuadro 7. Estimados de acumulación de biomasa seca en $\mathrm{kg} / \mathrm{ha}$ de pasto estrella (Cynodon nlemfuensis) por efecto de dosis crecientes de vermicompost durante cuatro años de evaluación. Turrialba, Costa Rica. 2003-2006.

\begin{tabular}{llllll}
\hline \multirow{2}{*}{$\begin{array}{c}\text { Trata- } \\
\text { mientos (t/ } \\
\text { vermicom- } \\
\text { post/ha) }\end{array}$} & $\mathbf{2 0 0 3}$ & $\mathbf{2 0 0 4}$ & $\mathbf{2 0 0 5}$ & $\mathbf{2 0 0 6}$ & Acumulado \\
\cline { 2 - 6 } & & & & & \\
\hline 0 & 2864,1 & 3439,2 & 2884,3 & 4220,0 & 10807,6 \\
$10-40$ & 2911,2 & 3092,1 & 2241,5 & 3015,1 & 11259,9 \\
$20-80$ & 2802,2 & 3693,0 & 3013,9 & 3995,2 & 11504,3 \\
\hline
\end{tabular}

dosis acumulada mayor al final del período de estudio (2006) (Cuadro 8).

A nivel de campo, no se encontró ningún efecto sobre otras variables físicas de suelo evaluadas, como fue el porcentaje de humedad gravimétrica y la densidad aparente del suelo.

Con base en los resultados obtenidos se puede concluir que el impacto del vermicompost utilizado, aunque en términos generales fue positivo, éste puede variar de acuerdo al tipo de suelo. El efecto sobre las propiedades químicas del suelo se observó especialmente con el uso de altas proporciones; pese a lo anterior no fue posible verificar este hecho a nivel de campo debido a la buena fertilidad del suelo utilizado. Aunque la diferencia no fue estadísticamente significativa, si se encontró una tendencia de incremento entre las dosis utilizadas en el campo.

Cuadro 8. Porcentaje de materia seca de pasto estrella $(C y$ nodon nlemfuensis) en respuesta a dosis crecientes de vermicompost a través de cuatro años de estudio. Turrialba, Costa Rica. 2003-2006.

\begin{tabular}{|c|c|c|c|c|}
\hline \multirow{2}{*}{$\begin{array}{c}\text { Tratamientos } \\
\text { (t vermicom- } \\
\text { post/ha) }\end{array}$} & \multicolumn{4}{|c|}{ Años de evaluación } \\
\hline & 2003 & 2004 & 2005 & 2006 \\
\hline 0 & $23,68 \mathrm{~ns}$ & $30,32 \mathrm{a} *$ & $31,86 \mathrm{ab}$ & $22,09 \mathrm{~b}$ \\
\hline $10-40$ & $22,84 \mathrm{~ns}$ & $27,40 \mathrm{ab}$ & $32,48 \mathrm{a}$ & $24,44 \mathrm{ab}$ \\
\hline $20-80$ & $21,13 \mathrm{~ns}$ & $24,86 \mathrm{~b}$ & $30,88 \mathrm{~b}$ & $24,95 \mathrm{a}$ \\
\hline
\end{tabular}

* Columnas con letras diferentes presentan diferencias significativas con la prueba Tuckey-Kramer HSD al 0,05 de probabilidad. Ns corresponde a diferencias no significativas.
Se concluye que el efecto del vermicompost orgánico en el suelo, así como su correspondiente respuesta en el crecimiento vegetal dependerá de las propiedades iniciales del suelo al cual se aplica la enmienda.

\section{AGRADECIMIENTOS}

Un agradecimiento especial a las y los estudiantes de la carrera de agronomía de la Sede del Atlántico de la Universidad de Costa Rica que a través de los años y en diferentes momentos del desarrollo de la investigación colaboraron como asistentes del proyecto en la toma de datos. A la Vicerrectoría de Investigación de la Universidad de Costa Rica por el apoyo en el financiamiento de este proyecto.

\section{LITERATURA CITADA}

Bertsch, F. 1998. La fertilidad de los suelos y su manejo. San José Costa Rica, Asociación Costarricense de la Ciencia del Suelo. $164 \mathrm{p}$.

Babou, OJ; Shiow-Long, T; Zeng, Y. 2007. Relationship between compost $\mathrm{pH}$ buffer capacity and $\mathrm{P}$ content on $\mathrm{P}$ availability in a virgin Ultisol. Soil Science 172:56-68.

Castellanos, J; Pratt, P. 1981. Mineralization of manure nitrogen-correlation with laboratory indexes. Soil Science Society of America Journal 45:354-357.

Castro, A; Henríquez, C; Bertsch, F. 2009. Capacidad de suministro de $\mathrm{N}, \mathrm{P}$ y K de cuatro abonos orgánicos. Agronomía Costarricense 33(1):31-43.

Chaimsohn, FP; Villalobos, E; Urpí, J. 2007. El fertilizante orgánico incrementa la producción de raíces de pejibaye (Bactris gasipaes K.). Agronomía Costarricense 31(2):31-43.

Durán, L; Henríquez, C. 2007. Caracterización química, física y microbiológica de vermicompostes producidos a partir de cinco sustratos orgánicos. Agronomía Costarricense 31(1):41-51.

Evanylo, G; Sherony, C; Spargo, J; Starner, D; Brosius, M; Haering, K. 2008. Soil and water environmental effects of fertilizer- manure-, and compost-based fertility practices in an organic vegetable cropping system. Agriculture, Ecosystems and Environment 127:50-58.

Hartz, T; Mitchell, J; Giagnnini, C. 2000. Nitrogen and carbon mineralization dynamics of manures and compost. HortScience 35(2):209-212. 
Henríquez, C; Cabalceta, G. 1999. Guía práctica para el estudio introductorio de los suelos con un enfoque agrícola. San José, Costa Rica Asociación Costarricense de la Ciencia del Suelo. 112 p.

Holdridge, SJ. 1978. Ecología basada en zonas de vida. San José, Costa Rica. IICA. 206 p.

Martínez, C. 1996. Potencial de la lombricultura. Elementos básicos para su desarrollo. Eds. A. Carballo y S. Bravo, Texcoco, México. 141 p.

Meléndez, G. 2003. Fracción orgánica del suelo: Residuos orgánicos y materia orgánica del suelo. In: G. Soto; G. Meléndez; L. Uribe. eds. Abonos orgánicos: Principios, aplicaciones e impacto en la agricultura. San José, Costa Rica. p. 1.

Narambuye, F; Haynes, R. 2007. Effect of organic amendments on soil $\mathrm{pH}$ and $\mathrm{Al}$ solubility and use of laboratory indices to predict their liming effect. Soil Science 171:748-754.

Schweizer, S; Vargas, A; Salas, E. 2003. Caracterización de diferentes compost utilizando técnicas físicas, químicas y biológicas. In: Soto, G; Descamps, P. eds. Memoria del I Encuentro Mesoamericano y del Caribe y III Encuentro Costarricense de Agricultores Experimentadores e investigadores en producción orgánica. Editorial del Norte, Costa Rica. p. 66-67.

Soto, G. 2003. Abonos orgánicos: Definiciones y procesos. In: Soto, G; Meléndez, G; Uribe, L. eds. Abonos orgánicos: Principios, aplicaciones e impacto en la agricultura. Centro de Investigaciones Agronómicas, Costa Rica. p. 27-33.

Soto, G; Meléndez, G. 2003. Indicadores químicos de calidad de abonos orgánicos. In: Soto, G; Meléndez, G; Uribe, L. eds. Abonos orgánicos: Principios, aplicaciones e impacto en la Agricultura. Centro de Investigaciones Agronómicas, Costa Rica. p. 59.

Sullivan, D; Miller, R. 2001. Compost quality attributes, measurements and variability. In: Stofella, P; Kahn, B. eds. Compost utilization in horticultural cropping systems. Lewis, USA. p. 108-112.

Wu, L; Martínez, C. 2000. Comparison of methods for evaluating stability and maturity of biosolids compost. Journal of Environmental Quality 29:424-429. 